\title{
LETTER
}

\section{Spontaneous and reflex head turning in brain death}

\author{
Yunfen $\mathrm{Wu}^{*}$ and Pedro Orizaola Balaguer
}

Head rotation in a patient with diagnosis of brain death (BD) has been scarcely reported. We report a new case, in order to highlight the importance of a correct identification of such movements to consider the legal implication revolving around the diagnosis of $\mathrm{BD}$.

A 42-year-old male mechanic was admitted to the ICU, after a 10-minute cardiopulmonary resuscitation for asystole following thoracic trauma. Left mydriasis and a Glasgow Coma Score of 3/15 were objectified. By 28 hours post arrest, brainstem reflexes disappeared, and profound cardiovascular instability developed 45 hours post arrest. Criteria for BD were fulfilled [1]. On day 5 post arrest, the electroencephalogram was isoelectric; the correlated video displayed images consisting of a slow turning movement of the patient's head from side to side after nociceptive stimuli, as well as spontaneously, with a persistence of seconds (see Additional file 1). Angiographic computed tomography documents the intracranial circulatory arrest, confirming BD.

The lateral head rotation arises primarily from activity of the spinal portion of the accessory (XI cranial) nerve and the cervical spinal cord (for example, sternocleidomastoid, trapezius and anterior vertebral muscles). The accessory nerve has a cranial and spinal component. The second to fourth spinal roots supply motor fibers to sternocleidomastoid and trapezius muscles. The anterior vertebral muscles receive innervation from the second to seventh cervical nerves.

In a BD patient, head turning movements have been described as a spontaneous automatism, as well as a reflex when induced after a stimulus [2-4]. In BD, the mechanisms underlying such movement remain obscure; nevertheless, it has been postulated that disconnection of the motor control corticospinal tracts may increase the excitability at the spinal level, and the cervical spinal cord may be a trigger of spontaneous movements in a hypoxic condition [5]. Further studies that clarify the characteristics of the involved generator will play a role in demonstrating these theories, confirming the spinal genesis of such complex movements.

\section{Additional file}

Additional file 1. A video showing the slow turning movement of the BD patient's head from side to side after nociceptive stimuli.

\section{Abbreviations}

$\mathrm{BD}$, brain death.

Competing interests

The authors declare that they have no competing interests.

\section{Acknowledgements}

The authors thank the Radiology Department for technical and analytical support with the angiographic computed tomography images. Written consent to publish was obtained from the patient's next of kin.

Published: 31 July 2013

References

1. Practice parameters for determining brain death in adults (summary statement). The Quality Standards Subcommittee of the American Academy of Neurology. Neurology 1995, 45:1012-1014.

2. Christie JM, O'Lenic TD, Cane RD: Head turning in brain death. J Clin Anesth 1996, 8:141-143.

3. Patterson KW, McShane: Reflex spinal cord activity as a cause of a delay in the diagnosis of brain death. Ir Med J 1991, 84:27-28.

4. Jain S, DeGeorgia M: Brain death-associated reflexes and automatisms. Neurocrit Care 2005, 3:122-126.

5. Saposnik G, Basile VS, Young GB: Movements in brain death: a systematic review. Can J Neurol Sci 2009, 36:154-160.

doi:10.1186/cc12753

Cite this article as: Wu Y, Balaguer PO: Spontaneous and reflex head turning in brain death. Critical Care 2013, 17:440. 
Additional files provided with this submission:

Additional file 1: 20130603_123033.mp4, 6614K

http://ccforum.com/imedia/7605627841009945/supp1.mp4 